\title{
Prediction and Perception of Technical Difficulties in Adult Cardiac Surgery
}

\author{
Yasser Y Hegazy, MD,${ }^{1,2}$ Mohamed S Nassar, MD,${ }^{2}$ Wael Hassanein, MD,${ }^{2}$ Mostafa Elhammami, MD, ${ }^{2}$ \\ Juergen Ennker, $\mathrm{MD}, \mathrm{PhD}^{1,3,4}$ \\ ${ }^{1}$ Department of Cardiac Surgery, Mediclin Herrzzentrum Lahr/ Baden, Germany, ${ }^{2}$ Department of Cardio-thoracic Surgery, \\ Faculty of Medicine, Alexandria University, Egypt, ${ }^{3}$ School of Medicine, Faculty of Health, University of Witten Herdecke, \\ Witten, Germany, ${ }^{4}$ Department of Cardiac Surgery, Klinikum Oldenburg, Germany
}

\section{ABSTRACT}

Background: Unexpected intra-operative technical difficulties are not uncommon in cardiac surgery. Our objective is to study the incidence, predictors, and consequences of unexpected difficulties in adult cardiac operations.

Methods: A total of 500 consecutive elective operations were included in the study. Before every operation, the surgeon and the assistant were asked to study the case and give a score (one to ten) for the expected technical difficulty of the operation. After every operation, the surgeon and the assistant were asked to give a score for the observed technical difficulty. The scores and perioperative data were collected and statistically analyzed.

Results: In relation to different interventions and consultant/trainee predictions, unexpected technical difficulties were encountered in $7 \%$ to $16 \%$ of cases. There was a significant difference between surgeons and trainees in the perception of level of increased difficulty, represented by the mean of differences between expected and observed score $(0.084 \pm$ 0.54 versus $0.016 \pm 0.5, P=.0002)$. In multivariable analysis, only female gender $(P<.0001)$ was identified as a factor associated with unexpected technical difficulties. There was no correlation between the incidence of complications and unexpected surgical difficulty. However, there was a weak positive correlation between operative times and observed difficulty score.

Conclusion: Unexpected technical difficulties are not uncommon in adult cardiac operations. Trainees tend to underestimate the difficulties perceived by the surgeon. This study can be a first step towards developing a technical difficulty score, which could be a helpful tool for medical quality management, as well as in training programs.

\section{INTRODUCTION}

With the progress made in adult cardiac surgery, surgeons are regularly faced with more complex cases. Preoperative assessment usually gives an idea of the expected

Received February 6, 2018; accepted June 1, 2018.

Correspondence: Yasser Hegazy, MD, Department of Cardiac Surgery, Mediclin Herzzentrum Labr, Hobbergweg 2, 77933 Lahr, Germany; +497821-9251019, fax: +497821-925-391000 (e-mail: yasserbegazy@yahoo.com). technical difficulties that the surgeon could face. However, facing unexpected intra-operative difficulties is not uncommon in our daily practice. While scores have been developed to predict patient risk and surgical outcome, the prediction of surgical difficulty is still highly subjective, and surgeon experience plays the main role in difficulty prediction and perception [Ad 2016]. What might be a difficult operation for one surgeon could be a relatively easy one for another. Moreover, an experienced surgeon might be able to deal with technical difficulties in a way that makes these difficulties go unnoticed by his less experienced assistant. Subsequently, it is common that young surgeons find most surgical tasks more difficult than expected when they perform them by themselves during their early practice.

The surgeon is not only expected to appreciate the level of difficulty of the surgical intervention, but is also expected to pass this experience to his trainees, who should develop this sense alongside the development of their technical skills. In the era where results are available to the public, centers are under continuous pressure to improve their results. When surgeons are faced with the dilemma of results versus training, the training programs could be affected. In this article, we try to shed the light on a surgeon's ability to predict and perceive technical difficulties in the modern era of cardiac surgery: a topic that, to our knowledge, has not been touched before, and can be a first step towards developing a technical difficulty score.

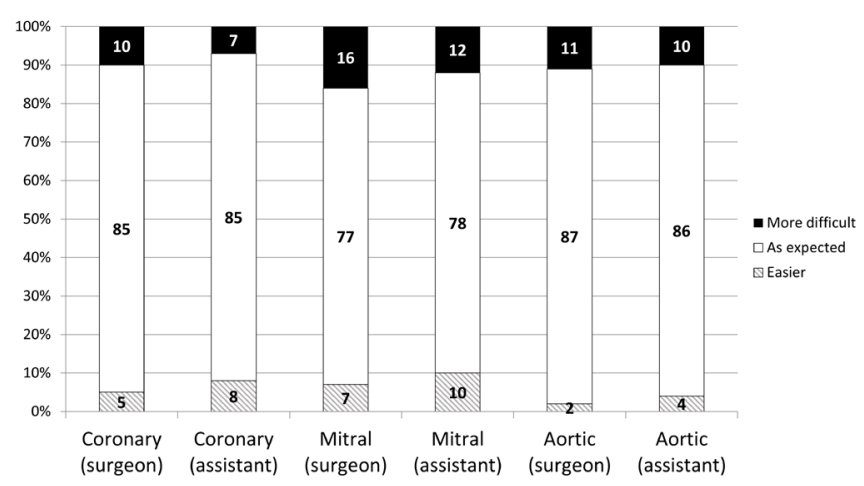

Figure 1. Observed technical difficulty compared to expected technical difficulty in different procedures. 
Table 1. Preoperative Data

\begin{tabular}{|c|c|c|c|c|}
\hline & All patients & Difficulty as expected (444 operations) & More difficult than expected ( 56 operations) & $P$ \\
\hline Female & $177(35.4 \%)$ & $143(32.21 \%)$ & $34(60.71 \%)$ & $<.0001$ \\
\hline Age & $59.66 \pm 15.2$ & $59.78 \pm 15.21$ & $58.7 \pm 15.27$ & .6191 \\
\hline Body Mass Index & $26.28 \pm 3.58$ & $26.25 \pm 3.55$ & $26.54 \pm 3.84$ & .5959 \\
\hline EuroSCORE II & $2.15 \pm 1.14$ & $2.15 \pm 1.13$ & $2.15 \pm 1.19$ & .9648 \\
\hline COPD & $26(5.2 \%)$ & $24(5.41 \%)$ & $2(3.57 \%)$ & .5602 \\
\hline Re-do & $20(4 \%)$ & $19(4.25 \%)$ & $1(1.79 \%)$ & .3695 \\
\hline
\end{tabular}

COPD indicates chronic obstructive pulmonary disease.

\section{METHODS}

This is a prospective dual-center study that was conducted between two Cardiothoracic Surgery departments. The study was part of a training program co-operation between the two centers. The Ethics Committees in both centers have waived the need for patient consent for the study.

The study included 500 unselected consecutive patients who had elective cardiac operations falling into one of three categories: coronary artery bypass graft (CABG), mitral valve procedures, or aortic valve procedures. All operations were performed through conventional mid-line sternotomy. Patients requiring concomitant procedures, apart from tricuspid valve repair for functional regurgitation, were not included in the study.

The patients' demographic characteristics, clinical details, and calculated EUROscore II scores were recorded. Before each operation, the surgeon and the assistant were asked to study the case and give a score for the expected technical difficulty of the operation. The lowest score was one (low difficulty), and the highest was ten (most difficult operation). Two consultant surgeons and one senior trainee in each unit took part in the study. The years of experience in cardiac surgery of the participating surgeons were 15, 20,34, and 38 years, and the years of experience of the participating trainees were six and eight years. A retired cardiac surgeon was asked to study all cases preoperatively and give his own score. After every operation, the surgeon and the assistant were asked to give a score for the observed technical difficulty. All participants gave their scores blinded from the others' scores.

Before starting the study, a small pilot study was conducted. Every participant was asked to give expected and observed scores for ten operations, and the study coordinator discussed the scores with each participant after every operation. The aim of the pilot study was to gain familiarity with the scoring system and to avoid outlier scores at the beginning of the study. The operations of the pilot study were not included in the current study.

If the observed difficulty score was higher than the expected difficulty score, the surgeon and/or trainee was asked to list the unexpected or more than expected difficulty that they faced during the procedure. The total operative times, cross clamp times, and cardiopulmonary bypass times were recorded. The recorded post-operative outcome included the duration of mechanical ventilation, ICU, and total hospital length of stay. Mortality and morbidity (bleeding, need for re-intervention, stroke, acute renal failure requiring dialysis and mediastinitis) were recorded.

\section{Statistical Analysis}

All data were collected using a standardized protocol. The statistical analysis was performed using JMP 5.1 software (SAS Institute, Inc, Cary, NC).

Continuous variables were expressed as mean \pm standard deviation (SD), and categorical variables were expressed as numbers and percentages. Continuous variables were compared using Student's t test. Tukey-Kramer HDS was used to compare all pairs. The fisher exact test and $\chi^{2}$ test were used for comparison of categorical variables. $P$ values less than .05 were considered statistically significant.

To study all factors associated with unexpected or more than expected technical difficulties, multiple regression models were calculated. Initially, 18 variables were studied. Further models were obtained by excluding the factors with $P$ values more than .25 in a stepwise manner. The final model was obtained when no further decrease in the Akaike Information Criterion (AIC) was observed (AIC= deviance of the model $+2 \times$ number of included parameters).

\section{Results}

Five hundred elective interventions were included in the study. The interventions were as follows: 320 CABGs (169 off-pump), 98 aortic valve replacements, and 82 mitral valve procedures (49 repairs and 33 replacements). Tricuspid valve repair for functional tricuspid regurgitation, associated with mitral disease, was carried out in 20 cases. The preoperative demographic data and important risk factors are shown in Table 1.

Fifty six cases $(11.2 \%)$ were marked by the operating surgeon as more difficult than expected, and 24 interventions $(4.8 \%)$ were described as easier than expected $(P=.075)$. In relation to different interventions and consultant/trainee predictions, unexpected technical difficulties were encountered 
Table 2. Events and Findings Perceived by the Surgeon and the Assistant as Unexpected or More Than Expected Difficulty

\begin{tabular}{|c|c|c|c|}
\hline \multirow[t]{3}{*}{ General } & Sternotomy & $3(0.6 \%)$ & $2(0.4 \%)$ \\
\hline & Adhesions & $6(1.2 \%)$ & $6(1.2 \%)$ \\
\hline & Cannulation and CPB & $2(0.4 \%)$ & $4(0.8 \%)$ \\
\hline \multirow{4}{*}{ CABG } & Intramuscular target & $8(2.5 \%)$ & $6(1.9 \%)$ \\
\hline & Calcified or severely atherosclerotic coronary wall & $14(4.4 \%)$ & $8(2.5 \%)$ \\
\hline & Bad graft material & $4(1.3 \%)$ & $5(1.6 \%$ \\
\hline & Calcified or atherosclerotic aortic wall & $2(0.6 \%)$ & $2(0.6 \%)$ \\
\hline \multirow{2}{*}{ Aortic valve } & Extensive valve calcification & $9(9.2 \%)$ & $7(7.1 \%)$ \\
\hline & Calcified or atherosclerotic aortic wall & $2(2 \%)$ & $2(2 \%)$ \\
\hline \multirow[t]{3}{*}{ Mitral Valve } & Difficult exposure & $13(15.9 \%)$ & $10(12.2 \%)$ \\
\hline & Extensive valve calcification & $2(2.4 \%)$ & $1(1.2 \%)$ \\
\hline & Left atrial thrombus & $1(1.2 \%)$ & 0 \\
\hline Total & & 88 (17.6\%) & $64(12.8 \%)$ \\
\hline
\end{tabular}

CPB indicates cardiopulmonary bypass; CABG, coronary artery bypass graft.

in $7 \%$ to $16 \%$ of cases (Figure 1). Only 10 interventions $(2 \%)$ were perceived by the operating surgeon as especially stressful situations. In the 56 operations identified as more difficult than expected, there were 88 findings that caused unexpected or more than expected technical difficulties (1.57 findings/operation). The details of the unexpected or more than expected technical difficulties are listed in table 2. The most commonly faced difficulties were small target vessels in CABG (5.9\%), extensive aortic valve calcification (9.2\%), and difficult exposure in mitral valve surgery (15.9\%).

There was no difference in pre-operative difficulty score between surgeons and trainees $(3.46 \pm 1.56$ versus $3.46 \pm$ $1.73, P=.9)$. The expert retired surgeon score matched three out of the four consultants' pre-operative scores (3.44 versus $3.46, P=.67 ; 3.21$ versus $3.23, P=.72$; and 3.32 versus 3.21 , $P=.07)$, and there was a significant difference between the expert score and one consultant (3.94 versus $3.77, P=.02$ ).

The post-operative scores given by the operating surgeon were significantly different from the pre-operative scores $(3.54 \pm 1.57$ versus $3.46 \pm 1.56$, respectively, $P=.0005)$, though the assistant scores were not different (postoperative $3.48 \pm 1.70$ versus preoperative $3.46 \pm 1.73$, respectively $P=.47)$. The distribution of preoperative and postoperative scores is shown in Figure 2.
When it comes to intra-operative difficulty perception by the trainees, the total number of operations perceived as more difficult than expected was significantly different from the number of operations perceived by the surgeons as more difficult than expected (42 [8.4\%] versus $56[11.2 \%], P<.0001)$. In addition, there was a significant difference between surgeons and trainees in the perception of the level of increased difficulty, represented by the mean of differences between expected and observed scores $(0.084 \pm 0.54$ versus $0.016 \pm 0.5, P=.0002)$.

To further study the effect of experience on the ability to predict technical difficulties, we compared the surgeons participating in the study with regard to the differences between their expected and observed difficulty scores. Despite the wide range of years of experience (15 to 38 years), there were no significant differences between them when comparing either all pairs (Tukey-Kramer HDS) or each pair (Student's t-test); with $P$ values of $.3929, .5512, .5703, .7636, .7526$ and .6943 .

Upon analysis of pre-operative characteristics, univariable analysis identified both female gender $(P<.001)$ and need for concomitant tricuspid intervention $(P=.045)$ as predictors of unexpected or more than expected technical difficulty. However, upon multivariable analysis, only female gender $(P<.0001)$ was identified. Euroscore II score was not correlated with unexpected or more than expected technical 
Table 3. Predictors of Unexpected or More than Expected Technical Difficulties

\begin{tabular}{lccc}
\hline Term & Estimate & Standard Error & $\mathrm{P}$ \\
\hline Intercept & 1.7265 & 1.7355 & .3198 \\
Female & 0.5861 & 0.1489 & $<.0001$ \\
Age & -0.0056 & 0.0114 & .6210 \\
Body Mass Index & -0.0215 & 0.0399 & .5906 \\
Re-do & -0.4484 & 0.5251 & .3932 \\
Diabetes Mellitus & 0.1096 & 0.1722 & .5246 \\
COPD & -0.0900 & 0.3853 & .8153 \\
EuroSCORE II & 0.0719 & 0.1329 & .5889 \\
Coronary bypass & 0.5660 & 0.5972 & .3432 \\
Aortic stenosis & 0.5842 & 0.5905 & .3225 \\
Aortic regurgitation & 0.2999 & 0.3772 & .4266 \\
Mitral stenosis & 0.4261 & 0.3659 & .2442 \\
Mitral regurgitation & 0.7585 & 0.5812 & .1919 \\
Tricuspid regurgitation & 0.1466 & 0.3683 & .6905 \\
\hline
\end{tabular}

COPD indicates chronic obstructive pulmonary disease.

difficulty $(P=.588)$ (Table 3$)$.

Recorded intra-operative times and post-operative complications are listed in table 4. There was no correlation between the incidence of mortality or complications and unexpected surgical difficulty. However, there was a positive, though weak, correlation between operative times (cross-clamp time, cardiopulmonary bypass time, and total operative time) and observed difficulty score (Table 5).

\section{DIsCussion}

In the current era of adult cardiac surgery, operative risk can be estimated with relatively acceptable accuracy [Ad 2016]. Pre-operative patient characteristics are entered into "apps" that come out with mortality/morbidity risk. However, there is poor correlation between the surgical risk and technical difficulty. For example, recent stroke, renal failure, poor myocardial function, and liver insufficiency are clear operative risks, but have little to do with the technical difficulties. On the other hand, a perfect complex mitral repair is technically more difficult, but is less risky, than mitral valve replacement.

In this study, we tried to analyze pre-operative characteristics to identify independent predictors of unexpected or more than expected technical difficulty. Only female gender came out as a strong predictor of unexpected or more than expected difficulty, a result which matches the consensus that females have a small aortic root, a small left atrium, and small coronary vessels [Hiteshi 2014; Buellesfeld 2013; Nikitin 2003]. Age and Euroscore II, on the other hand, were not predictors of unexpected/more than expected difficulty.
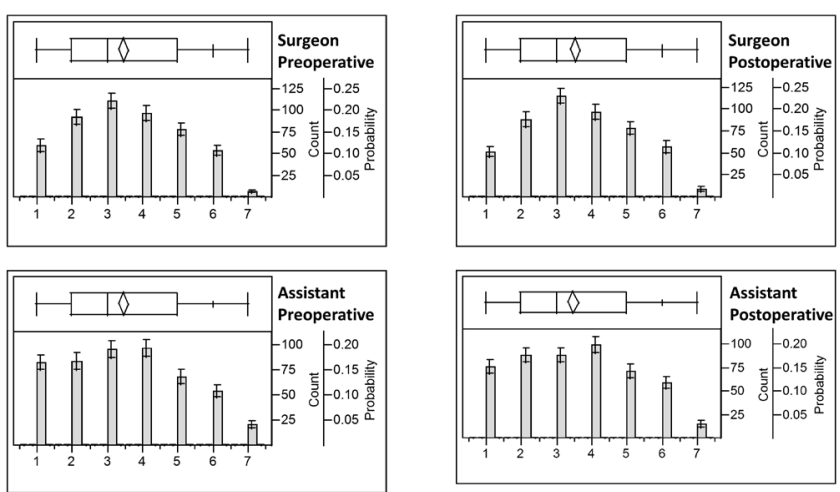

Figure 2. Distribution of preoperative and postoperative technical difficulty scores.

Surgical planning depends on the surgeon's analysis of available clinical and diagnostic information. With modern imaging technology, the surgeon is expected to have enough data to predict the difficult situations that he might face during the intervention, with little influence from personal experience. This was clear in our results, where pre-operative estimation of technical difficulty was not different between expert, consultant, or senior trainee. However, with all these advances, prediction is still not completely accurate, and a significant difference is seen when a surgeon is asked to rescore the difficulty after procedure: more cases were labelled difficult rather than easy $(11.2 \%$ versus $4.8 \%, P=.075)$. What was also interesting was the difference in the perception of difficulty level between the consultant/main surgeon and the assistant/trainee.

Threat perception involves the recognition of aspects of the situation that pose a threat to the safety of the situation. It remains the first step of risk management, upon which assessment and action will rely [Pauley 2011]. Trainees in their last year of training are expected to have reached a level of technical and non-technical skills that enables them to start their own career. In our study, trainees could recognize unexpected intra-operative difficulty, but not at the same level of perception as the main surgeon. This could be partially attributed to the difference in experience: a trainee could be distracted by the theater environment, the assistant's situational awareness could be reduced by a lack of effective communication, or a form of actor-observer bias could occur [Pauley 2011; Diwadkar 2010; Jones 1976].

Cardiothoracic surgery training programs are currently under pressure. With centers' results constantly under review, and restrictions on working hours, trainees have a relatively decreased chance to develop their technical and cognitive skills [Connors 2009]. Simulation training has been introduced into cardiac surgery in the last few years, along with special curriculum that addresses the uncommon adverse events which would challenge a newly starting consultant who might have not faced similar situations across his traditional training program. Though neither of the two centers included in the study have simulation programs implemented in training, the reported results from other centers are encouraging: 
Table 4. Mean Recorded Times and Incidence of Complications in the Studied Cohort

\begin{tabular}{|c|c|c|c|c|}
\hline Operative time ( $\mathrm{min}$ ) & $224.67 \pm 47.21$ & $223.34 \pm 46.54$ & $235.23 \pm 51.47$ & .1044 \\
\hline CPB (min.) & $97.80 \pm 32.41$ & $95.11 \pm 29.04$ & $118.58 \pm 47.04$ & .0046 \\
\hline Cross clamp (min.) & $69.58 \pm 22.07$ & $68.07 \pm 20.34$ & $81.24 \pm 30.37$ & .0129 \\
\hline Ventilation (hours) & $13.32 \pm 40.28$ & $13.81 \pm 42.52$ & $9.39 \pm 11.82$ & .0857 \\
\hline Hospital stay (days) & $9.01 \pm 2.62$ & $8.97 \pm 2.55$ & $9.29 \pm 3.18$ & .4682 \\
\hline Mortality & $15(3 \%)$ & $13(2.93 \%)$ & $2(3.57 \%)$ & .7902 \\
\hline Bleeding $(\mathrm{ml})$ & $846.65 \pm 422.83$ & $842.79 \pm 420.47$ & $877.23 \pm 443.88$ & .5839 \\
\hline Re-exploration & $33(6.6 \%)$ & $29(6.53 \%)$ & $4(7.14 \%)$ & .8622 \\
\hline Stroke & $6(1.2 \%)$ & $5(1.13 \%)$ & $1(1.79 \%)$ & .6693 \\
\hline
\end{tabular}

CPB indicates cardiopulmonary bypass; ICU, intensive care unit.

feedback from both trainees and seniors report improved operating room familiarity and comfort after implementation of the program [Mokadam 2017]. However, these simulation programs cannot be a replacement for traditional operative room experience [Odell 2015].

In all traditional programs, trainees are expected to start their technical experience through relatively straightforward cases. Assigning a case to a trainee usually depends on how technically challenging this case might be. In our field, this usually depends on consultant experience and available clinical data. Unexpected difficulty may force the consultant to switch places across the table, and subsequently, the trainee will lose a case he was very keen to do. A technical difficulty score can be very useful in training programs. The development of a score can help case selection not only for trainees, but also for consultants at different levels of experience.

The need for a difficulty index has been recently noted by other surgical specialties as a map for building surgical skills [Ban 2014; Osborne 2006]. Unfortunately, in cardiac surgery, the only available scores are calculated risk scores such as Euroscore and STS risk score, which cannot be used to predict technical difficulties. In the current study, the commonly used risk assessment scores, represented by Euroscore II, were not correlated to the difficulty level scored by the surgeon, which was a strongly predicted finding. The risk score does not take into account the presence of a small aortic root, extensive valve calcification, a small left atrium, or small target vessels, all of which are labelled as technically challenging anatomy. On the other hand, the subjective technical difficulty score has failed to predict any post-operative complications, which are more accurately predicted by risk scores [Ad 2016].

Developing a technical difficulty score is not only important for the training program, but also for resource management and patient satisfaction. A technically difficult operation is likely to take more time than an easy one. This lengthened time has a direct effect on the operative cost. In addition, an operation that takes longer than expected might lead to postponing the next planned operation, and this can negatively influence the satisfaction of the patient.

The technical difficulty score needed for training programs and for medical quality management has to be a standardized, objective score. In our opinion, the first step towards developing such an objective difficulty score is to create and study subjective scores, like what was done in this study. Subjective technical difficulty scores are also important for the assessment of new techniques. New techniques can prove to have clear advantages in comparison with standard ones. However, many of these techniques remain underused. A good example of this is multiple arterial revascularization. A possible explanation for the slow adoption of new, and clearly superior techniques, is the fear of technical difficulty, even if it is not associated with increased adverse effects. The availability of evidence regarding subjective difficulty scores and their correlation with the learning curve would encourage surgeons to adopt these techniques, since they can expect approximately how many cases are needed to overcome the perceived technical difficulties. In addition, surgeons can document subjective scores for the observed technical difficulties and include these scores in their own database to use them to monitor the development of their skills when starting new techniques.

\section{Limitations}

The number of patients in this study, though not small, was not sufficient to develop an objective technical difficulty 
Table 5. Correlation Between Operative Times and Observed Difficulty Score

\begin{tabular}{lccc}
\hline & Cross-clamp time & Cardiopulmonary bypass time & Total operative time \\
\hline Orthogonal fit ratio & 190.4 & 410.7 & 905.7 \\
Correlation & 0.172 & 0.22 & 0.126 \\
Intercept & 17.85 & 21.82 & 118.01 \\
Slope & 13.8 & 20.82 & 30.1 \\
Lower CL & 6.66 & 11.98 & 12.8 \\
Upper CL & 28.61 & 34.28 & 70.76 \\
\hline
\end{tabular}

$\mathrm{CL}$ indicates confidence limit.

score. Moreover, echocardiography and angiography data were not included in the statistical analysis, which would be essential for developing such a score. Another limitation is that we looked into prediction and perception of difficulty, but not into decision making in response to situations. In this field, experience would play a bigger role and influence the effect of unexpected difficulties on the operative outcome.

\section{CONCLUSION}

In the current era, unexpected or more than expected technical difficulties remain a challenge, and it is not uncommon that even the most experienced surgeons have to face them. Only female gender was identified as an independent predictor for unexpected or more than expected technical difficulty. The trainees tend to underestimate the difficulties perceived by the surgeon. A currently used risk score, Euroscore II, does not predict unexpected technical difficulties. Further studies are required to develop an objective technical difficulty score, which could be a helpful tool for medical quality management, as well as in managing training programs to better assign cases according to experience level.

\section{REFERENCES}

Ad N, Holmes SD, Patel J, et al. 2016. Comparison of EuroSCORE II, original EuroSCORE, and the society of thoracic surgeons risk score in cardiac surgery patients. Ann Thorac Surg 102:573-9.

Ban D, Tanabe M, Ito H, et al. 2014. A novel difficulty scoring system for laparoscopic liver resection. J Hepatobiliary Pancreat Sci 21:745-53.

Buellesfeld L, Stortecky S, Kalesan B, et al. 2013. Aortic root dimensions among patients with severe aortic stenosis undergoing transcatheter aortic valve replacement. JACC Cardiovasc Interv 6:72-83.

Connors RC, Doty JR, Bull DA, et al. 2009. Effect of work-hour restriction on operative experience in cardiothoracic surgical residency training. J Thorac Cardiovasc Surg 137:710-13.

Diwadkar GB, Jelovsek JF. 2010. Measuring surgical trainee perceptions to assess the operating room educational environment. J Surg Educ 67:210-16.

Hiteshi AK, Li D, Gao Y, et al. 2014. Gender differences in coronary artery diameter are not related to body habitus or left ventricular mass. Clin Cardiol 37:605-9.

Jones EE. 1976. How do people perceive the causes of behavior? Experiments based on attribution theory offer some insights into how actors and observers differ in viewing the causal structure of their social world. American Scientist 64:300-5.

Mokadam NA, Fann JI, Hicks GL, et al. 2017. Experience with the cardiac surgery simulation curriculum: Results of the resident and faculty survey. Ann Thorac Surg 103:322-8.

Nikitin NP, Witte KK, Thackray SD, et al. 2003. Effect of age and sex on left atrial morphology and function. Eur J Echocardiogr 4:36-42.

Odell DD, Macke RA, Tchantchaleishvili V, el al. 2015. Resident perception of technical skills education and preparation for independent practice. Ann Thorac Surg 100:2305-13.

Osborne SA, Severn P, Bunce CV, et al. 2006. The use of a pre-operative scoring system for the prediction of phacoemulsification case difficulty and the selection of appropriate cases to be performed by trainees. BMC Ophthalmol 6:38.

Pauley K, Flin R, Yule S, et al. 2011. Surgeons' intraoperative decision making and risk management. Am J Surg 202:375-81. 